\title{
The Potential Use of Urinary CtDNA Profiling in the Treatment of Breast Cancer
}

\author{
Nel Ivonne* and Aktas Bahriye \\ Department of Gynecology, Medical Center, University of Leipzig, Germany
}

*Corresponding author: Nel Ivonne, Department of Gynecology, Medical Center, University of Leipzig, Germany.
Received Date: July 23, 2020

Published Date: August 20, 2020

\begin{abstract}
Cell-free circulating tumor DNA (ctDNA), shed into the blood stream by apoptotic or necrotic tumor cells of either primary or metastatic sites, became an extensively investigated and very promising analyte in oncology research. If passing through the kidney barrier, ctDNA is likely to occur in urine. Literature research revealed a lack of studies aiming at diagnostic use of urinary ctDNA. Most studies investigating urinary ctDNA were performed in the field of urological cancers emphasizing, however, that urinary liquid biopsies were suitable to draw conclusive real time pictures of ctDNA alterations coming from circulation and hence strengthen the hypothesis that genetic profiling of urinary ctDNA could be valuable to gain tumor-related information also in other solid tumors such as breast cancer. Usually, clinical treatment decisions are based on mutation profiles that were received from initial tissue biopsies. Though, during therapy the genetic tumor profile might change e.g. gain and loss of genetic alterations that might be relevant for targeted therapy options or treatment resistance. Particularly patients with advanced breast cancer may acquire mutations during treatment cycles and might benefit from serial ctDNA sequencing to find new targetable mutations and gain access to tailored therapy. Here, the use of urinary ctDNA might offer an opportunity for non-invasive longitudinal genotyping and testing for actionable mutations. In contrast to plasma-derived ctDNA, only a few studies were performed using urinary ctDNA from patients with breast cancer and revealed that targeted NGS appeared to be a sensitive method to detect tumor-specific genetic features. In this mini review we sought to illuminate the potential use of urinary ctDNA for longitudinal disease monitoring at frequent intervals and low effort for patients with breast cancer.
\end{abstract}

Keywords: Breast cancer; Mutation profiling; Urinary ctDNA; NGS; Targeted therapy; Disease monitoring; Recurrence; Resistance

Abbreviations: cfDNA: cell free circulating DNA; ctDNA: cell free circulating tumor DNA; CNV: Copy Number Variation; SNV: Single Nucleotide Variant; ER: Estrogen Receptor; PR: Progesterone Receptor; HER2: Human Epidermal growth factor Receptor-2; ddPCR: digital droplet PCR; NGS: Next Generation Sequencing; PIK3CA: Phosphatidylinositol-4,5-Bisphosphate 3-Kinase Catalytic Subunit Alpha; TP53: Tumor protein p53; CDH1: Cadherin-1; MLL3: Myeloid/lymphoid or mixed-lineage leukemia protein 3; ESR1: Estrogen Receptor 1; BRCA 1/2: Breast Cancer 1/2; DTC: Disseminated Tumor Cell

\section{Introduction}

Cell-free circulating DNA (cfDNA) became an extensively investigated and very promising analyte in oncology research. Particularly, DNA fragments originating from the tumor cells, socalled circulating tumor DNA (ctDNA), appear to be surrogates of the primary tumor or metastatic sites thereof. Usually, clinical treatment decisions are based on mutation profiles that were received from initial tissue biopsies. Therapy, however, might alter the genetic tumor profile and cause the occurrence of genetic changes that could be relevant for targeted therapy options or treatment resistance. In breast cancer, genetic profiling of ctDNA from blood plasma was shown to have good potential for clinical use and might help to find individual treatment options and to monitor metastatic relapse. Cancer recurrence is of high relevance for both, patients diagnosed with early breast cancer and pre- 
treated women with advanced breast cancer. Due to limited therapy options, particularly, women diagnosed with the aggressive triple negative breast cancer subtype might benefit from mutation profiling using non-invasive tools allowing disease monitoring at frequent intervals. Here, the utility of urinary ctDNA might offer promising opportunities to obtain non-invasive liquid biopsies and make access to tailored individual treatment choices possible.

\section{About Cell Free Circulating Tumor DNA (ctDNA)}

Cell free circulating tumor (ctDNA) is shed into the blood stream by apoptotic or necrotic tumor cells. Characteristic features of apoptosis are DNA fragmentation and formation of apoptotic bodies. Consequently, the release of short nuclear DNA fragments of about $167 \mathrm{bp}$ was shown to have a lower molecular weight compared to DNA fragments released by necrotic cells, thus, making it possible to determine tumor-derived DNA based on size distribution [1,2]. More precise approaches to identify ctDNA originating from tumor cells are based on the molecular alterations of tumor-specific genetic features like DNA mutations, methylation and copy number variations (CNVs) [3]. If circulating cell free DNA in the blood is passing through the kidney barrier it is likely to be found in urine and also called trans renal DNA or ucfDNA [46]. Urinary cfDNA might also originate from apoptotic or necrotic cells coming in direct contact with urine such as cells from the genitourinary tract. Since glomerular filtration, which takes place in the pores of the glomerular barrier, works like separation by size, only small DNA fragments with a size of about $100 \mathrm{bp}$ are able to pass and might appear in urine. Molecular analysis of urinary ctDNA could be useful to gain tumor-related information not only in patients with urological cancers but also with other solid tumors such as breast cancer $[4,7]$. The concentration of ctDNA extracted from body fluids like blood plasma or urine might vary hugely depending on numerous patient-individual parameters such as disease stage, treatment response and further physiological and patho-logical conditions as well as the body fluid itself. Circulating ctDNA in blood can be detected in serum and plasma, with the latter one containing up to 20-fold higher concentrations [8]. Hence, a variety of ctDNA extraction and detection methods were developed [9]. At the time being there is no standard protocol for isolation and detection of urinary cell free DNA, but plenty of techniques for isolation of low-molecular weight DNA fragments are available and appear to be sensitive and reproducible $[10,11]$. Literature research revealed a lack of studies aiming at diagnostic use of ucfDNA. Most publications described preliminary results based on small patient cohorts. Novel molecular technologies such as targeted next generation sequencing (NGS) or digital droplet PCR (ddPCR) are offering promising opportunities for the translation of ucfDNA based tumor profiling into the clinic though [2]. Most studies investigating urinary cfDNA were performed in the field of urological cancers including renal, bladder and prostate cancer emphasizing that urinary liquid biopsies were suitable to draw conclusive real time pictures of DNA alterations coming from circulation. At this point it appears noteworthy that a close similarity was described between bladder cancer and breast cancer [12] . Based on genomic expression and mutation analyses it was shown that bladder cancer was, similar to breast cancer, distinguishable into luminal and basal tumors and that those molecular subtypes appeared to be of prognostic relevance [13].

\section{Molecular Features of Breast Cancer Tissue}

Breast cancer is a very heterogeneous disease and can be classified into distinct molecular subtypes. Luminal A type tumors are hormone-receptor positive (estrogen-receptor (ER) and/or progesterone-receptor (PR) positive), human-epidermal-growthfactor-receptor-2 (HER2) negative and show low levels of the protein Ki-67, meaning low proliferation. Usually, they are of low are lowgrade, tend to grow slowly and patients have the best prognosis. The majority of patients is diagnosed with ER positive tumors and might receive endocrine therapy. Luminal B type tumors are also hormone-receptor positive (ER and/or PR positive), and either HER2 positive or HER2 negative, with high levels of Ki-67 meaning that they grow faster. The patient's prognosis is slightly worse compared to luminal A. Patients suffering from HER2 (also called ERBB2) amplified tumors are prone to treatment with targeted therapy such as trastuzumab or pertuzumab. Triple negative breast cancer (TNBC) is hormone-receptor negative (neither ER nor PR) and HER2 negative with poor prognosis. The majority of TNBCs are of high grade and show an aggressive phenotype. Patients usually receive chemotherapy but have an increased risk of recurrence [14]. Molecular analysis of the breast cancer subtypes revealed that luminal A tumors, although being associated with good prognosis and therapy response, were presenting the majority of driver mutations such as PIK3CA $45 \%$, GATA3 14\%, MAP3K1 $\sim 13 \%$, TP53 12\%, CDH1 9\% [15]. In contrast, triple negative tumors appeared to lack driver mutations (PIK3CA 9\%, MLL3 $\sim 5 \%$ ), but showed mutations of the tumor suppressor gene TP53 in $80 \%$ of the cases.

\section{Somatic and Targetable Mutations in Breast Cancer}

As mentioned above mutation profiling of breast cancer tissue revealed distinct molecular subtypes presenting characteristic somatic mutations, including single nucleotide variants (SNVs) and copy number variations (CNVs). Next to a huge number of low frequency variants, the usual suspects among mutated genes in breast cancer are TP53 and PIK3CA. In addition, to broaden the understanding of tumor initiation, promotion and progression it is of high medical relevance to identify and investigate somatic mutations that drive the cancer phenotype in order to find possible therapies targeted against the products of these abnormal genomic alterations. Currently, the genes ESR1 and HER2 are not only 
characteristic features of the respective molecular subtype, in fact they serve as therapeutic targets for endocrine and antibody-based therapeutics such as tamoxifen and trastuzumab or pertuzumab. Further, ESR1 mutations were found to be associated with tamoxifen resistance [16]. Among TNBC a variety of subgroups has been identified including high cellular proliferation, increased immunological infiltrate, basal-like and mesenchymal phenotype as well as deficiency in homologous recombination which was partly associated with loss of BRCA1 or BRCA2 function [17]. Although challenging, the molecular analysis of TNBC gave rise to potential options for tailored therapy strategies such as modified chemotherapy approaches targeting the DNA damage response, angiogenesis inhibitors, immune checkpoint inhibitors, or even anti-androgens, all of which are currently being evaluated in phase I to III clinical studies [17].

\section{Liquid Biopsy Based on ctDNA vs. Initial Tissue Biopsy}

Since ctDNA was postulated to be shed by all tumor sites, even micro metastases, it could enable early detection of recurrence and also identification of molecular alterations during treatment courses e.g. gain and loss of genetic changes that might be relevant for targeted therapy options or treatment resistance. Usually, clinical treatment decisions are based on mutation profiles that were received from initial tissue biopsies. During therapy, however, the genetic tumor profile might change meaning that distinct mutations such as targetable driver mutations might become functionally neutral passenger mutations. Consequently, treatment efficacy might be affected ultimately leading to resistance. A study by Rothe, et al. used next-generation sequencing (NGS) evaluation on 50 cancer genes covering 2,800 mutations listed in the Catalogue of Somatic Mutations In Cancer (COSMIC) in 60 tumor tissues and 31 plasma samples from 17 metastatic breast cancer patients and was able to show a $76 \%$ concordance rate between tissue and plasma. The results strengthen the hypothesis that ctDNA from plasma can be prospectively tested as an alternative to metastatic biopsies [18] Another aspect favoring liquid biopsy sampling is clonal selection that might take place during treatment courses and could causing the occurrence of targetable mutations that were not detectable in the primary tumor tissue. Particularly patients with advanced breast cancer may acquire mutations during treatment cycles and might benefit from serial ctDNA sequencing to find new targetable mutations and gain access to tailored therapy. Patients receiving aromatase inhibitors often become resistant due to acquired ESR1 mutations. Keup, et al. evaluated cfDNA variants in 40 hormone receptor-positive and HER2-negative patients with metastatic breast cancer and found genetic variants of MUC16, BRCA2, ERBB3, and AR in $>90 \%$ of the cases [19]. The authors reported one particular case in which longitudinal monitoring revealed an increase of pathogenic and likely pathogenic PIK3CA and ESR1 variant allele frequency under everolimus and exemestane, 8 months before therapy failure was confirmed by visual staging during clinical follow-up. Here, the use of urinary ctDNA might offer an opportunity for non-invasive longitudinal genotyping and testing for actionable mutations such as ESR1, HER2 or AKT1.

\section{Analysis of ctDNA}

Quantitative levels of cfDNA concentrations alone were shown to be insufficient for successful disease monitoring or cancer detection [20]. One promising approach to identify tumor specific alterations in cfDNA might be sequencing of cancer-related mutations in the primary tumor tissue and verifying them in cfDNA at baseline. For analyzing tumor-specific ctDNA a variety of molecular methods is available. Common and reliable methods for mutational analysis are digital droplet PCR (ddPCR) or the more costly next generation sequencing (NGS). Many studies compared mutational profiles in plasma-derived ctDNA and matched tissue samples from breast cancer patients and found good correlations. In a study by Oshiro et al, serum samples were collected preoperatively from 313 stage I-III breast cancer patients. Samples from patients with PIK3CA mutant tumors $(n=110)$ were analyzed using ddPCR and $22.7 \%$ were found to be positive. No PIK3CA mutant ctDNA was detected in the serum samples of 50 healthy women and 30 breast cancer patients with PIK3CA non-mutant tumors. The patients with PIK3CA mutant ctDNA were then divided into ctDNAhigh and ctDNAlow groups. Statistical analysis revealed that that ctDNAhigh but not ctDNAlow status appeared to be a significant and independent prognostic factor for primary breast cancer patients [21]. Interestingly, in patients with early stage TNBC, simply the presence of ctDNA after NACT, was reported to be predictive for recurrence [22,23]. As proof of concept, Page et al evaluated targeted NGS of cfDNA for analysis of mutations and amplification in 16 genes in 42 patients with metastatic breast cancer compared to 9 healthy controls [24]. On average, $89 \%$ of the 158 targeted regions were covered at $>500 x$. No mutations or CNVs were detected in cfDNA of the healthy controls. ESR1, TP53, and PIK3CA were found to be the top 3 mutated genes in cfDNA. Further, they performed serial monitoring in 9 patients and demonstrated that cfDNA profiling of mutations and amplifications could provide useful information regarding tumor heterogeneity, clonal evolution, and response to treatment. Their data confirmed that the targeted NGS approach had potential clinical utility. Remarkably, $21 \%$ of the patients might have benefit from an alternative therapy if ctDNA monitoring was translated into clinical routine. Occurrence of ERBB2 amplification in plasma ctDNA could guide a switch towards anti-HER2 therapy, and emergence of ESR1 mutations could indicate that the patient might profit from a treatment change away from endocrine therapy to standard chemotherapy [24]. 


\section{Urinary vs. Plasma ctDNA in Breast Cancer}

In contrast to plasma-derived ctDNA, only a few studies were performed using urinary ctDNA from patients with breast cancer. Focusing on the PIK3CA mutation, Liu et al investigated correlations between ctDNA derived from plasma vs. urine of 200 patients with early breast cancer [25]. They reported a lower mean concentration of cfDNA extracted from urine compared to plasma with a strong correlation though. They also compared urinary cfDNA of breast cancer patients and healthy volunteers and found significantly elevated cfDNA levels in the patient's group. Their results emphasize that urinary ctDNA allows close correlation to the molecular profile of the tumor and even might predict relapse. Recently, a study by Guan et al described the utility of urinary ctDNA to monitor recurrence in 300 patients with early breast cancer using serial sampling before treatment and at various time points during follow up [26]. They found detectable mutations in 38\% of the patients and the agreement with matched tissue samples was $97 \%$. Patients with positive mutations were associated with greater risk of relapse. Considering poor prognosis and limited treatment options of patients diagnosed with basal-like breast cancer it is of particularly high clinical relevance to identify driver mutations a well as targetable genetic alterations. Additionally, non-invasive molecular monitoring tools at high frequencies are urgently needed. Here, longitudinal mutation profiling using urinary ctDNA might offer a promising approach.

\section{Combined Approach for Risk Stratification: DTCs and ctDNA}

For patients diagnosed with primary breast cancer at early stages risk stratification is difficult as imaging methods might not be sensitive enough to detect secondary tumor sites or micro metastases. Despite successful treatment of the primary tumor recurrence occurs in about $30 \%$ of breast cancer patients. One reason might be hematogenous spread during early disease stages [27]. Disseminated breast cancer cells preferentially migrate into the bone marrow (BM) where they become dormant. Due to low proliferation in this "steady state" disseminated tumor cells (DTCs) are persistent against systemic chemotherapy and may cause metastatic relapse at a later stage [28]. DTCs may serve as independent prognostic markers that are associated with impaired survival [29]. One attempt might be the detection of DTCs in the bone marrow, which is a rather unusual body fluid to be routinely tested, but which can be aspirated during primary surgery [30]. DTCs in the bone marrow can specifically be stained and visualized as described elsewhere [31]. Breast cancer patients with positive DTC status were associated with elevated risk for relapse [27]. Hence, DTC-positive patients might benefit from non-invasive high frequency genetic profiling using ctDNA to monitor treatment response and cancer recurrence. A combination of initial bone marrow puncture and subsequent longitudinal genetic analyses using urinary and plasma-derived ctDNA in an alternating pattern might be a promising approach for early detection of cancer relapse.

\section{Tumor Derived Urinary Exosomes}

Another promising analyte that draw increased researches attention are so-called exosomes which are membrane-derived extracellular nanovesicles of about 30-100 $\mathrm{nm}$ released by several types of cells [32]. Exosomes were detected in numerous body fluids, including blood and urine and play a crucial role in tumor biology. Tumor cells have been shown to produce and secrete higher quantities of exosomes compared to normal cells [33]. Exosomes are known to play a crucial role in carcinogenesis, proliferation, formation of pre-metastatic niche, angiogenesis, metastasis, and chemoresistance [34]. Communication with the tumor microenvironment and the ability to exchange information by secreting growth factors, cytokines, chemokines, and small molecular mediators such as nucleotides makes them valuable cellto-cell messengers. Not only might they contain genetic information useful for diagnostic purposes or disease monitoring, engineered exosomes could also function as cargo containing specific molecules that can affect the recipient cell thus leading to targeted therapy [32]. Exosomes released by tumor cells are affecting the tumor microenvironment by transferring oncogenes and nucleic acids. Thus, they participate in tumor progression and metastasis [35]. Interestingly, it was reported that bone-marrow derived cells might be recruited to tumor and pre-tumor tissue by exosomes. About primary breast cancer, a recent pilot study by Ando et al reported a novel screening approach based on urinary exosomes. In a small cohort consisting of 22 patients and 26 healthy controls they revealed that the combined expression levels of miR-21 and MMP-1/CD63 in urinary exosomes could detect 95\% of early BC without metastasis [36]. Although the study lacks statistical power, it demonstrated a certainly simple screening approach.

\section{Advantages of Urinary ctDNA Profiling}

Urine-based tests are non-invasive and therefore very patientfriendly. They are at most convenience for the patients as they could collect their samples at home and ship them to clinical laboratories. Novel preservative reagents were developed to stabilize cfDNA in urine samples during storage and transportation for up to 7 days at various temperatures up to $37^{\circ} \mathrm{C}$ [37-39].There would be no need for frequent visits in the outpatient unit, which could save time and cost, for both, patient and clinic. Neither special equipment nor trained medical staff would be required for sampling. Urinary ctDNA could be used for longitudinal disease monitoring at frequent intervals and low effort. Increased sample volumes could compensate relatively low quantities of urinary ctDNA. Urinary ctDNA analysis are advantageous for patients living in remote areas, because it enables them to be monitored closely without traveling into the clinic as for blood sampling. 


\section{Conclusion}

Urine-based liquid biopsies allow truly non-invasive and costeffective sampling and offer a promising opportunity to monitor therapy response or tumor progression at high frequencies. Mutational profiling of urinary ctDNA might be prognostic for cancer relapse and could help to guide personalized treatment options and thus offering the most effective treatment for patients with breast cancer. Considering the aggressive tumor growth and increased resistance towards available chemotherapeutic options patients with triple negative tumors might particularly benefit from high frequency serial mutation profiling based on ctDNA from both blood and urine in an alternating manner.

\section{Acknowledgement}

We would like to thank Prof. Sabine Kasimir-Bauer for her consistent collaboration, scientific support and always friendly advice.

\section{Conflict of Interest}

Authors declare no conflict of interest.

\section{References}

1. Jahr S, Hentze H, Englisch S, Hardt D, Fackelmayer FO, et al. (2001) DNA fragments in the blood plasma of cancer patients: quantitations and evidence for their origin from apoptotic and necrotic cells. Cancer Res 61(4): 1659-1665.

2. Salvi S, Gurioli G, Giorgi U de, Conteduca V, Tedaldi G, et al. (2016) Cellfree DNA as a diagnostic marker for cancer: current insights. Onco Targets Ther 9: 6549-6559.

3. Frenel JS, Carreira S, Goodall J, Roda D, Perez-Lopez R, et al. (2015) Serial Next-Generation Sequencing of Circulating Cell-Free DNA Evaluating Tumor Clone Response to Molecularly Targeted Drug Administration. Clin Cancer Res 21(20): 4586-4596.

4. Botezatu I, Serdyuk O, Potapova G, Shelepov V, Alechina R, et al. (2000) Genetic analysis of DNA excreted in urine: a new approach for detecting specific genomic DNA sequences from cells dying in an organism. Clin Chem 46(8 Pt 1): 1078-1084

5. Lichtenstein AV, Melkonyan HS, Tomei LD, Umansky SR (2001) Circulating nucleic acids and apoptosis. Ann N Y Acad Sci. 945: 239-249.

6. Lu T, Li J (2017) Clinical applications of urinary cell-free DNA in cancer: current insights and promising future. Am J Cancer Res. 7(11): 23182332 .

7. Su Y-H, Wang M, Brenner DE, Ng A, Melkonyan H, et al. (2004) Human Urine Contains Small, 150 to 250 Nucleotide-Sized, Soluble DNA Derived from the Circulation and May Be Useful in the Detection of Colorectal Cancer. J Mol Diagn 6(2): 101-107.

8. Lee TH, Montalvo L, Chrebtow V, Busch MP (2001) Quantitation of genomic DNA in plasma and serum samples: higher concentrations of genomic DNA found in serum than in plasma. Transfusion 41(2): 276282.

9. Heitzer E, Ulz P, Geigl JB (2015) Circulating tumor DNA as a liquid biopsy for cancer. Clin Chem 61(1): 112-123.

10. Lee EY, Lee E-J, Yoon H, Lee DH, Kim KH (2020) Comparison of Four Commercial Kits for Isolation of Urinary Cell-Free DNA and Sample Storage Conditions. Diagnostics (Basel) 10(4): 234.

11. Streleckiene G, Reid HM, Arnold N, Bauerschlag D, Forster M (2018) Quantifying cell free DNA in urine: comparison between commercial kits, impact of gender and inter-individual variation. Biotechniques 64(5): 225-230.

12. Wirtz RM, Fritz V, Stöhr R, Hartmann A (2016) Molecular classification of bladder cancer. Possible similarities to breast cancer. Pathologe 37(1): 52-60.

13. Damrauer JS, Hoadley KA, Chism DD, Fan C, Tiganelli CJ, et al. (2014) Intrinsic subtypes of high-grade bladder cancer reflect the hallmarks of breast cancer biology. Proc Natl Acad Sci U S A 111(8): 3110-3115.

14. Marotti JD, Abreu FB de, Wells WA, Tsongalis GJ (2017) Triple-Negative Breast Cancer: Next-Generation Sequencing for Target Identification. Am J Pathol 187(10):2133-2138.

15. Cancer Genome Atlas Network (2012) Comprehensive molecular portraits of human breast tumours. Nature 490(7418): 61-70.

16. Li S, Shen D, Shao J, Crowder R, Liu W, et al. (2013) Endocrine-therapyresistant ESR1 variants revealed by genomic characterization of breastcancer-derived xenografts. Cell Rep 4(6): 1116-1130

17. Denkert C, Liedtke C, Tutt A, Minckwitz G von (2017) Molecular alterations in triple-negative breast cancer-the road to new treatment strategies. The Lancet 389(10087): 2430-2442.

18. Rothé F, Laes J-F, Lambrechts D, Smeets D, Vincent D, et al. (2014) Plasma circulating tumor DNA as an alternative to metastatic biopsies for mutational analysis in breast cancer. Ann Oncol 25(10): 1959-1965.

19. Keup C, Benyaa K, Hauch S, Sprenger-Haussels M, Tewes M, et al. (2020) Targeted deep sequencing revealed variants in cell-free DNA of hormone receptor-positive metastatic breast cancer patients. Cell Mol Life Sci 77(3): 497-509.

20. Schwarzenbach H, Hoon DSB, Pantel K (2011) Cell-free nucleic acids as biomarkers in cancer patients. Nat Rev Cancer 11(6): 426-437.

21. Oshiro C, Kagara N, Naoi Y, Shimoda M, Shimomura A, et al. (2015) PIK3CA mutations in serum DNA are predictive of recurrence in primary breast cancer patients. Breast Cancer Res Treat 150(2): 299-307.

22. Radovich M, Jiang G, Hancock BA, Chitambar C, Nanda R, et al. (2020) Association of Circulating Tumor DNA and Circulating Tumor Cells After Neoadjuvant Chemotherapy with Disease Recurrence in Patients with Triple-Negative Breast Cancer: Preplanned Secondary Analysis of the BRE12-158 Randomized Clinical Trial. JAMA Oncol: e202295.

23. Garcia-Murillas I, Chopra N, Comino-Méndez I, Beaney M, Tovey H, et al. (2019) Assessment of Molecular Relapse Detection in Early-Stage Breast Cancer. JAMA Oncol 5(10): 1473-1478.

24. Page K, Guttery DS, Fernandez-Garcia D, Hills A, Hastings RK, et al. (2017) Next Generation Sequencing of Circulating Cell-Free DNA for Evaluating Mutations and Gene Amplification in Metastatic Breast Cancer. Clin Chem 63(2): 532-541.

25. Liu Z, Liu W (2018) Association of urinary and plasma DNA in early breast cancer patients and its links to disease relapse. Clin Transl Oncol 20(8): 1053-1060.

26. Guan G, Wang Y, Sun Q, Wang L, Xie F, et al. (2020) Utility of urinary ctDNA to monitoring minimal residual disease in early breast cancer patients. Cancer Biomark 28: 111-119.

27. Braun S, Vogl FD, Naume B, Janni W, Osborne MP, et al. (2005) A pooled analysis of bone marrow micrometastasis in breast cancer. $\mathrm{N}$ Engl J Med 353(8): 793-802.

28. Pantel K, Brakenhoff RH, Brandt B (2008) Detection, clinical relevance and specific biological properties of disseminating tumour cells. Nat Rev Cancer 8(5): 329-340.

29. Banys-Paluchowski M, Fehm T, Janni W, Solomayer E-F, Hartkopf A (2019) Circulating and Disseminated Tumor Cells in Breast Carcinoma: Report from the Consensus Conference on Tumor Cell Dissemination during the $3^{\text {th }}$ Annual Meeting of the German Society of Senology, Berlin, 27 June 2019. Geburtshilfe Frauenheilkd 79(12): 1320-1327. 
30. Hoffmann O, Aktas B, Goldnau C, Heubner M, Oberhoff C, et al. (2011) Effect of ibandronate on disseminated tumor cells in the bone marrow of patients with primary breast cancer: a pilot study. Anticancer Res 31(10): 3623-3628.

31. (2020) $16^{\text {th }}$ Leipzig Research Festival for Life Sciences. Schaefer uaM, (edt), Leipzig: LIFE Forschungszentrum, Universität Leipzig, Germany, p.83.

32. Rashed MH, Bayraktar E, Helal GK, Abd-Ellah MF, Amero P, et al. (2017) Exosomes: From Garbage Bins to Promising Therapeutic Targets. Int Mol Sci 18(3): 538.

33. Jenjaroenpun P, Kremenska Y, Nair VM, Kremenskoy M, Joseph B, et al. (2013) Characterization of RNA in exosomes secreted by human breast cancer cell lines using next-generation sequencing. PeerJ 1: e201.

34. Taverna S, Giusti I, D’Ascenzo S, Pizzorno L, Dolo V (2020) Breast Cancer Derived Extracellular Vesicles in Bone Metastasis Induction and Their Clinical Implications as Biomarkers. Int J Mol Sci 21(10): 3573.

35. Anastasiadou E, Slack FJ (2014) Cancer. Malicious exosomes. Science 346(6216): 1459-1460.
36. Ando W, Kikuchi K, Uematsu T, Yokomori H, Takaki T, et al. (2019) Novel breast cancer screening: combined expression of miR-21 and MMP-1 in urinary exosomes detects $95 \%$ of breast cancer without metastasis. Sci Rep 9: 13595 .

37. (2020) Research and Development Division, Streck, Inc., Omaha, NE 68128, USA. Stabilization of cfDNA in Urine Using a Preservative Reagent During Sample Processing, Transport, and Storage.

38. Li Z, Tang H, Zhang T, Liu C, Zhu S, et al. (2019) Abstract 2236: Development and validation of urine cfDNA preservative kit for detection of genomic alterations in cancer. In: Proceedings: AACR Annual Meeting 2019; Atlanta, GA: American Association for Cancer Research; 07012019 . p. 2236.

39. Mehta A, Meers N, Pasmans D, Vankerckhoven V, den Bossche RV, et al. (2020) Collection, storage and transport recommendations for first-void urine samples. 